\title{
Avaliação do uso de lâmpadas LED: Estudo de caso em um Serviço de Nutrição e Dietética em Natal - RN
}

\author{
Evaluation of the use of LED lamps: Case study in a Nutrition and Dietetics Service in Natal - RN \\ Evaluación del uso de lámparas LED: Estudio de caso en un Servicio de Nutrición y Dietética en \\ Natal - RN
}

Juliana Bianca Maia Franco

ORCID: https://orcid.org/0000-0002-7566-2688 Universidade Potiguar, Brasil

E-mail: juliana_franco_@hotmail.com

Laís Karla da Silva Barreto

ORCID: https://orcid.org/0000-0002-5267-5355 Universidade Potiguar, Brasil E-mail: laisbarreto@gmail.com

Hilderline Câmara de Oliveira ORCID: https://orcid.org/0000-0003-4810-117X Universidade Potiguar, Brasil

E-mail: hilderlinec@hotmail.com

Tarciara Magley da Fonseca Pereira ORCID: https://orcid.org/0000-0002-7570-8471

Universidade Potiguar, Brasil E-mail: tarciara@ufersa.edu.br

Ismael de Mendonça Azevedo ORCID: https://orcid.org/0000-0002-1695-3522 Universidade Potiguar, Brasil

E-mail: ismaeldemendonca@bol.com.br

Fábio da Silva

ORCID: https://orcid.org/0000-0003-0039-0128 Universidade Potiguar, Brasil

E-mail: fabiosoyme@hotmail.com

Heitor César Costa Oliveira

ORCID: https://orcid.org/0000-0003-4778-6613

Universidade Potiguar, Brasil

E-mail: cartoriosbrasil@gmail.com

Luana Araújo Matos

ORCID: https://orcid.org/0000-0003-0396-2312

Universidade Potiguar, Brasil

E-mail: matosluana@hotmail.com

\begin{abstract}
Resumo
A estudo referiu-se de um estudo de caso, com a mira de discernir os prováveis resultados ecossistêmicos com a transferência de luminárias fluorescentes por luminárias LED na Unidade de Alimentação e Nutrição do Hospital Universitário Onofre Lopes, a manar do dimensionamento comparativo das fontes de luminárias. Para instruir a coleta de dados, foi feita uma inspeção a unidade de alimentação, em que em todo espaço foi feita a descrição de luminárias que havia trabalhando e marca a extensão do clima. Diante dos benefícios, percebeu-se que a desigualdade do LED em analogia com luminárias fluorescentes compactas é o inferior fluxo luminoso difundido, que é perto da metade do fluxo luminoso difundido comumente por luminárias fluorescentes. Os benefícios das luminárias LED são seu parcel impacto ecossistêmico e sua enorme vitalidade profícuo. Não obstante, para que isso transcorra, devem ser instalados condições diminutos para a estirpe de luminosidade do LED. Porque embora do evento de que os excelentes artefatos respeitam às utilidades visuais humanas, isto não é considerado no comércio como um completamente. Dessa forma, pôde-se argumentar que as habilidades luminosas apresentadas pelos LEDs são imediatas às trombadas para luminárias fluorescentes compactas. A transferência de um artefato por outro, torna fundamental a diagnóstico caso a caso. É iminente descobrir habilidades para LED inferior dos economias médios achados para fluorescentes compactas, o que não concede que se confirme que o LED é impreterivelmente realmente mais eficaz.
\end{abstract}

Palavras-chave: Impacto ambiental; Iluminação; LED; Serviço de nutrição e dietética. 


\begin{abstract}
The research dealt with is a case study to know the possible environmental impacts by replacing fluorescent lamps with LED lamps in the University Hospital of the Food and Nutrition Unit Onofre Lopes, from the comparative dimensions of the types of lamps. To start the data collection was made a visit to feed unit, where in each sector was done counting lamps was working and the area measured in square meters. Before the results, it was noticed that the disadvantage of LED compared to CFLs is low lumen output, which is about half the luminous flux emitted by fluorescent lamps normally. The advantages of LED lamps is their low environmental impact and long service life. But for this to happen should be established minimum requirements for the quality of LED lighting. For despite the fact that the best products meet the human visual needs, this is not observed in the market as a whole. Thus, it could be concluded that the luminous efficiencies presented by the LEDs are close to those found in compact fluorescent bulbs. The substitution of one product for another, makes it necessary to analyze each case. You can find LED efficiencies below the mean values for compact fluorescent, which does not allow the affirmation that the LED is necessarily always more efficient.
\end{abstract}

Keywords: Environmental impact; Lighting; LED; Nutrition and dietary service.

\title{
Resumen
}

La investigación fue un estudio de caso, con el objetivo de conocer los posibles impactos ambientales de la sustitución de lámparas fluorescentes por lámparas LED en la Unidad de Alimentación y Nutrición del Hospital Universitario Onofre Lopes, a partir del dimensionamiento comparativo de los tipos de lámparas. Para iniciar la recolección de datos, se realizó una visita a la unidad de potencia, donde se contó el número de lámparas que estaban funcionando en cada sector y se midió la superficie del ambiente. Dados los resultados, se observó que la desventaja del LED en comparación con las lámparas fluorescentes compactas es el bajo flujo luminoso emitido, que es aproximadamente la mitad del flujo luminoso emitido normalmente por las lámparas fluorescentes. Las ventajas de las lámparas LED son su bajo impacto medioambiental y su larga vida útil. Pero para que eso suceda, se deben establecer requisitos mínimos para la calidad de la iluminación LED. Porque a pesar de que los mejores productos satisfacen las necesidades visuales humanas, esto no se observa en el mercado en su conjunto. Así, se podría concluir que las eficiencias luminosas que presentan los LED son cercanas a las encontradas para las lámparas fluorescentes compactas. La sustitución de un producto por otro obliga a analizarlo caso por caso. Es posible encontrar eficiencias para los LED por debajo de los valores promedio encontrados para los fluorescentes compactos, lo que no nos permite afirmar que los LED sean necesariamente siempre más eficientes.

Palabras clave: Impacto ambiental; Encendiendo; LED; Servicio de nutrición y dietética.

\section{Introdução}

Segundo Teixeira et al. (2018), o planejamento físico das unidades de alimentação é um trabalho de natureza abrangente, envolvendo além de direcionamento das áreas, outros fatores, como a ambiência do trabalho. Esta é considerada o conjunto de elementos envolventes que condicionam as atividades administrativas e operacionais e determinam em grande parte a qualidade e quantidade de trabalho produzido.

De acordo com a Resolução RDC nº 216 da Agência Nacional de Vigilância Sanitária (ANVISA) "a luminosidade de uma Unidade de Alimentação e Nutrição (UAN) deve proporcionar a visualização de forma que as atividades sejam realizadas sem comprometer a higiene e as características sensoriais dos alimentos. As luminárias localizadas sobre a área de preparação dos alimentos devem ser apropriadas e estar protegidas contraexplosão e quedas acidentais”.

Cury (2020) diz que o layout corresponde ao arranjo dos diversos postos de trabalho nos espaços existentes na organização, envolvendo além da preocupação de melhor adaptar as pessoas ao ambiente de trabalho, segundo a natureza da atividade desempenhada, a arrumação dos móveis, máquinas, equipamentos e matérias primas. Correspondendo assim, a distribuição física de elementos em um ambiente ou espaço, com intuito de atender as indigências do local, contribuindo assim para o ambiente organizacional, aproveitando espaços e reduzindo custos.

Teixeira (2018) ainda relata que a iluminação exerce uma forte influência no comportamento das pessoas. A utilização adequada evita doenças visuais, aumenta a eficiência no trabalho e diminui o número de acidentes. Essa poderá ser distribuída de forma uniforme, evitando sombras, ofuscamentos, contrastes, reflexos fortes. Sendo colocada de forma a incidir numa direção que não prejudique os movimentos ou a visão dos trabalhadores.

Pinto (2018) afirma que as primeiras lâmpadas comercializadas foram as incandescentes. O princípio de 
funcionamento dessas lâmpadas é baseado no aquecimento de um filamento de tungstênio através de passagem de corrente elétrica. Enquanto houver corrente elétrica circulando pelos terminais da lâmpada, esse filamento se mantém aquecido tornando-se incandescente. Devido ao fato de emitir luz através do aquecimento, semelhante ao processo realizado pelo sol, a luminária incandescente apresenta algumas vantagens, como o alto índice de representação de cores, refletindo assim a cópia fiel do que está sendo iluminado, assim como acontece no caso da iluminação natural.

Ainda segundo Pinto (2018), as luminárias fluorescentes apresentam tempo de vida e eficácia luminosa superiores quando comparadas com as incandescentes. Não produzem calor excessivo (lâmpadas frias) e sua temperatura de cor (luz branca) geralmente é elevada, o que estimula atividade física, sendo assim uma grande vantagem. Uma desvantagem dessas lâmpadas é a presença de gases, promovendo efeitos prejudiciais ao meio ambiente quando descartadas de forma inadequada.

Aas lâmpadas LED (do inglês Light Emission Diode, diodo emissor de luz) são um dispositivo eletrônico semicondutor que quando polarizado diretamente, dentro do semicondutor ocorre à recombinação de lacunas e elétrons. Sendo capaz de produzir muito mais luz visível do que calor, tornando-as mais eficientes, chegando a economizar 50\% da energia comparada às fontes tradicionais.

Souza (2015) diz que os aparelhos de LED requerem menos energia para operar e, portanto, geram pouco calor. As luzes do LED podem durar mais de 1000 horas, enquanto as alógenas duram no máximo 100 horas. Também, como o aparelho não usa filtros nem bulbos que são degradados quando são usados na luz convencional, a intensidade de luz não diminui conforme seu uso.

Por isso, Nóbrega (2020) ilustra vários fatores que geram impactos positivos no meio ambiente com o uso de luminárias LED. Alguns desses são relacionados com a iluminação, como a possibilidade de consumir forma reduzida a energia elétrica e diminuição de resíduos tóxicos.

Estudos da Agência Internacional de Energia (IEA) apontam que o iluminamento representa 19\% dos gastos com energia elétrica em todo o mundo. No Brasil, este valor é de 24\%, sendo distribuído em: $35 \%$ para aplicações residenciais, $41 \%$ para aplicações comerciais, $19 \%$ para aplicações públicas e 5\% para aplicações industriais.

Por fim, Corazza (2018) quando conceitua gestão ambiental, afirma que esta envolve planejamento, organização, orientando a empresa a alcançar metas [ambientais] específicas. Um aspecto relevante da gestão ambiental é que sua introdução requer decisões nos níveis mais elevados da administração e, portanto, envia uma clara mensagem à organização de que se trata de um compromisso corporativo. Gestão ambiental pode se tornar também um importante instrumento para as organizações em suas relações com consumidores, o público em geral.

\section{Metodologia}

A pesquisa tratou-se de pesquisa quantitativa do tipo descritiva, sendo um estudo de caso (Yin, 2015; Estrela 2018; Pereira et al, 2018) com o objetivo de analisar a compensação nos impactos ambientais com a permuta de luminárias fluorescentes por lâmpadas LED na Unidade de Alimentação e Nutrição do Hospital Universitário Onofre Lopes, pressupondo o controle preventivo de danos ambientais. Uma vez constatado o perigo ao meio ambiente, deve-se ponderar sobre os meios de evitar ou minimizar o prejuízo.

Para iniciar a coleta de dados, foi feita uma visita a unidade de alimentação, onde em cada setor foi realizada a contagem de lâmpadas que estavam funcionando e medida sua superfície.

Para o cálculo do quantitativo de lâmpadas necessário para iluminar cada ambiente, utiliza-se a expressão a seguir, a qual mostra que o fluxo luminoso é encontrado por:

$$
\emptyset=\frac{\mathrm{E} \times 1,25 \times \mathrm{A}}{\eta}
$$


onde:

$\emptyset=$ Fluxo Luminoso (em lúmen);

$\mathrm{E}=$ Iluminamento (em Lux);

1,25 = Usado para compensar a perda do fluxo luminoso;

$\mathrm{A}=$ Superfície a ser iluminada $\left(\mathrm{em} \mathrm{m}^{2}\right)$;

$\eta=$ Eficiência de distribuição do fluxo luminoso sobre a área.

Para verificar a eficácia luminosa dos produtos LED de marcas e modelos mais deparados no mercado brasileiro, realizou-se a pesquisa em catálogos deparados em endereços eletrônicos.

No meio de os produtos LED acessíveis, atendendo a apenas luminárias tipo bulbo e tubulares que são empregadas para iluminação global em construções, é exequível descobrir eficiências luminosas similares às achadas em luminárias fluorescentes. Além disso, os seus parâmetros de estirpe de iluminação, avaliados pela temperatura de cor e índice de reprodução de cores, também são destacados.

É fundamental acentuar que a tecnologia LED está em fase de crescimento e espera-se que no breve período alcançará as totais eficiências luminosas acessíveis no comércio. Já é iminente conseguir no comércio brasílico uma diversidade de artefatos LED que podem permutar luminárias incandescentes e fluorescentes.

A consulta em registros dos essenciais fabricantes de LED no comércio brasilense indica eficácias luminosas, em sua gigante excelência, na faixa de 50 a 110 lumens/Watt, por isso, fez-se uma média aritmética desses padrões e se resolveu 85 lumens/Watt, concorde demonstra-se no Quadro 1.

Quadro 1 - Eficiência de lâmpadas.

\begin{tabular}{|l|l|}
\hline LÂMPADA & EFICIÊNCIA \\
\hline Incandescente & 10 lumens/Watt \\
\hline Fluorescente & 50 lumens/Watt \\
\hline LED & 85 lumens/Watt \\
\hline
\end{tabular}

Fonte: Autores.

Em processo localizar-se a número de lâmpadas LED essenciais para cada extensão. Conquanto, vale acentuar que os artefatos pesquisados sucederam dos fabricantes Philips, Osram, LG e Samsung, estando estes os mais achados no comércio. Os objetos indicados para esse diagnóstico se cercearam às luminárias tipo bulbo e tubulares, usadas para iluminação global em construções. Objetos propostos à luminosidade para apresentação de objetos comerciais, iluminamento pública, luminosidade de fachadas, lâmpadas industriais, entre outros, não são analisados neste trabalho. A amostra comporta os produtos LED classificados mais meritórios para a permutação de luminárias incandescentes e fluorescentes compactas, o que, no entanto, não caracteriza todos os artefatos acessíveis no mercado brasileiro.

A consulta em registros permite a análise da analogia entre fluxo luminoso e eficiência luminosa. As integrais eficiências são achadas nas lâmpadas LED tubulares, frequentemente destinadas à permutação de fluorescentes compactas tubulares. Estes fundamentos difundem um alto fluxo luminoso. A global eficiência descoberta foi de 113,7 lumens/Watt. No caso das luminárias LED tipo bulbo, a maior eficiência descoberta foi de 81 lumens/Watt. Por isso, que se resolveu a transferência de luminárias fluorescentes por luminárias LED de $80 \mathrm{~W}$. 


\section{Resultados e Discussão}

Para salvaguardar $100 \%$ de conformidade de luminosidade de uma Unidade de Alimentação e Nutrição (UAN) do Hospital Universitário Onofre Lopes, asseverando a visualização de forma que os afazeres sejam cumpridos sem abalar a salubridade e as propriedades sensoriais das comidas, são imprescindíveis 249 luminárias fluorescentes de vigor $40 \mathrm{~W}$ e meramente 85 luminárias LED de vigor de $80 \mathrm{~W}$, perto de $66 \%$ a aquém a quantidade primordial.

A última Apuração de Posse de Equipamentos e Hábitos de Uso, desenrolada entre os momentos de 2004 e 2006 pelo PROCEL (ELETROBRÁS, 2017), informa que, para o campo residencial, a luminosidade conta cerca de $14 \%$ da demanda integral de energia elétrica nas moradias brasílicas. No setor mercantil, a luminosidade corresponde por $22 \%$ da demanda e no âmbito público por 23\%. Estes elementos evidenciam a magnitude de que a ascensão do emprego de energia seja associada pela rendimento energético dos produtos propostos à luminosidade.

O Departamento de Energia dos Estados Unidos (2016) também pondera a inteligência LED como a caminho mais acessível para a transferência das fluorescentes compactas e investe em desenvolvimento e inserção do artefato no comércio. Segundo o Programa Ambiental das Nações Unidas (2015), a crucial fonte de luminosidade empregue em edificações em todo o campo é a lâmpada incandescente. Essas luminárias apontam reduções eficiências luminosas e breve fase de vida, estimulando um gigante impacto ambiental. Faz-se crucial, para compensar a debate progressivo por energia, a transferência das marcas de luminárias incapazes por tipos cada vez mais competentes.

As lâmpadas LED são usualmente elaboradas de lâmpadas LED integradas juntamente drivers e dissipadores de chama marchetados em uma modelo singular. Para as luminárias LED, especificamente do tipo bulbo, é trivial situar-se baixos índices de fluxo luminoso.

No entanto de mostrarem habilidades luminosas similares, o uso de LED inclusive pode ser auspicioso se forem destacados todos os impactos ambientais e a vida útil do artefato. O Departamento de Energia dos Estados Unidos, provou que os impactos ambientais de luminárias LED vistas em 2017 no comércio eram cerca de $20 \%$ menores que os impactos causados por luminárias fluorescentes compactas. Desta forma, se faz primordial a informação dos cidadãos quanto à magnitude do emprego desta inteligência correspondente às suas conveniências em nexo à longevidade, competência energética e pequeno impacto ambiental. Argumentos esses de florescente importância da coletividade vigente que mira a dever de defender o meio ambiente.

Os artefatos LED aconselham a superioridade de não empregarem elementos tóxicos em sua disposição, como o mercúrio constante em luminárias fluorescentes compactas. A construção da inteligência LED é expressa, e as capacidades amplificam a todo dia. Ao passo que o Departamento de Energia dos Estados Unidos, "existem dois recursos para engendrar luz branca com LED: por meio de da transformação com fósforo e atravessadamente da aglutinação de distintas cores".

Estes haveres intercedem na competência luminosa dos artefatos, bem como a temperatura de cor e demais numerosos elementos. Repara-se conforme a obtenção da luz branca através da combinação de cores (CC) ou conversão com fósforo (F), e a temperatura de cor divergem os artefatos em analogia às suas eficiências. São ditas condições ardentes essas abaixo de 3000 $\mathrm{K}$, e condições frias, acima de $5000 \mathrm{~K}$. Espera-se que as diferenciais entre efeitos luminosas devidas à temperatura de cor caiam sucessivamente. Espera-se também, que luminárias LED compreendam eficiências luminosas maiores que $200 \mathrm{~lm} / \mathrm{W}$ até 2015.

A Comissão de Energia da Califórnia concebeu há pouco tempo um plano de lei para a estirpe da iluminação para LED, que daria repercussão na Califórnia meramente, bem como referência para a adição do artigo no comércio. A meta é suscitar a discussão e a apoderação de ação objetivando não recomeçar os similares erros executados quando da transferência de incandescentes por fluorescentes. As lâmpadas fluorescentes dispuseram questões de consideração na sua fase de divulgação no comércio por problemas de habilitação da cor e vida útil. As referências foram facilidades a partir de uma 
pesquisa elaborada pelo Pacific Northwest National Laboratoty.

Persevera a pressão empregada pelos financiadores para que a manufatura seja distribuída e vista resultados com destino a o investidor. O Departamento de Energia dos Estados Unidos (2012) é um gigante investidor na apuração, construção e lançamento no comércio da engenharia LED, suportando os negócios por meio de das especificações de reinvestimento acerca de a percepção das distribuidoras de energia. Ele consideração que até 2030 as luminárias e iluminação LED equivalerão a 70\% do reserva americano, em lúmens-hora gastos. Isto garantiria $67 \%$ de racionamento de energia na esfera de iluminadura, em analogia a um panorama sem LED. A norma filantropa preparada pela Universidade da Califórnia conferência com o plano Energy Star, presente em total a nação.

O Programa Ambiental das Nações Unidas (2015) mira para o episódio de que a lei da estirpe e da competência dos artefatos deva ser ocorrência de perfil harmônica internacionalmente. Ele concede que as nações validem particularidades diminutos de habilitação para os artefatos, não obstante sugere que os objetivos e padrões de estirpe compitam discutidos no meio de nações fabricantes e importadores internacionalmente, para que nutram reciprocidade.

Para luminárias fluorescentes, contam hodiernamente 48 regulamentações de estirpe com sequela pátrio em várias nações, cada qual com miras e limites sui generis e constantemente cruzando em combate. No entanto disso, mais de $80 \%$ das luminárias fluorescentes são elaboradas na China, país em que a jurisprudência não é assistida por uma inspeção forte. Por conseguinte, a legislatura em cada nação importador tem unicamente objetivo disfarçado sobre os itens. Como estas legislaturas são heterogêneos, fica deveras mais atribulado para os fabricantes seguirem aos dispares particularidades.

Para seguir a sui generis normas, os fabricantes admitiriam mais gastos acerca de os custos de montagem sobre tamanho, que poderiam chegar a 5\% do custo global. Inclusive igualmente, inclusive atualmente não conta uma lei mundial para luminárias fluorescentes copiosamente consente. Realizações como a Asia Lighting Compact, International CFL Harmonization Iniciative, US/EC Cooperation, 4E SSL Research e SEAD têm por mira descobrir anuência e articulação entre as condições de predicado e performance em sui generis nações, mas até o momento isto não é veracidade. Sucede que ao aquém os dispositivos para teste em laboratório são consoantes entre a superioridade das leis para lâmpadas fluorescentes, respaldando-se em gigante ideia nas decisões da Comissão Eletrotécnica Internacional.

A competência da luminosidade LED consegue tocar alavancada com a apoio de limitações de automatização. Empresas como Philips, Marvell e Bridgelux apresentam-se progredindo objetos com drivers Wi-Fi, que consentem que as lâmpadas LED se encontrem controladas em tempos remotos. Peças como este já contam no comércio, mas ainda assim têm salientes custos para os clientes.

A esperança é de que esta engenharia seja empregada previamente no campo mercantil, em que a perspectiva de atenuar o emprego de energia em gigante classificação é cativante. Estas luzes têm a prerrogativa de admitirem o comando do coração, vigor da luminosidade e a mensuração do uso de energia.

Por conclusão, os inconvenientes pertinentes à estirpe da luminosidade necessitam estar priorizados e esclarecidos previamente da sua saída e antes da instalação de planos de competência energética. Recomenda-se ainda que a lei seja feita de modelo harmônica entre pátrias exportadores e importadores, para que não se encontrem choques que impeçam o acolhimento de sui generis questões.

\section{Conclusão}

Pode-se concluir que as habilidades luminosas apresentadas pelos LEDs são ulteriores às trombadas para luminárias fluorescentes compactas. A transferência de um gênero por outrem ainda assim é ponderosa, sendo fundamental a diagnóstico caso a caso. Logo, inclusive é iminente descobrir eficiências para LED inferior das economias médias descobertas contra fluorescentes compactas, o que não deixa que se confirme que o LED é impreterivelmente constantemente mais competente. 
A troca das luminárias fluorescentes modelo tubulares por luminárias de led modelo tubulares na unidade de alimentação e nutrição viria diminuir a número de luminárias, descontaria o custo com energia elétrica e por conseguinte essa troca atenuaria os impactos ambientais prejudiciais presentes nessa unidade.

O fluxo luminoso lançado do LED é comumente inferior se contraposto ao fluxo luminoso lançado por fluorescentes, em que se trata de luminárias perfil bulbo. Isso importa que em uma transferência de um gênero por outro seja fundamental a inauguração de mais pontos de luz para se conseguir o mesmo fluxo luminoso. A predicado de iluminação do LED é pertinente aos fundamentos de conforto humano e análogo à categoria das fluorescentes compactas, quando ponderados os artefatos de superior estirpe.

Isto informa que é iminente que o LED liquide as precisões visuais humanas, no entanto não indica que todos os artefatos do comércio inteirem esta categoria, sendo fundamental normatização de Pressupostos ínfimos de qualidade da luminosidade, excepcionalmente o monitoramento do IRC, temperatura de cor, vida útil, dimerização e direcionalidade do facho de luz, transportando então os artefatos estandardizados.

Para que não aconteçam executados os similares desvios executados ao passo que do acréscimo das luminárias fluorescentes no comércio, que tardaram sua consideração pelos clientes, recomendações são praticadas. No meio de distintas termos, recomenda-se que nesta conjunção os artefatos LED compitam direcionados a comércios de nicho e que não aconteçam executados planeamentos de rendimento energética antes que questões de estirpe sejam definidos.

Em frente disso, recomenda-se a articulação das disposições de estirpe entre nações exportadores e importadores a plano mundial. Isso abstém-se que a disparidade de condições atravanque a produção de artefatos, que deteriam que seguir a completamente as sui generis questões.

\section{Referências}

Agência Internacional de Energia. (2019). Light's Labour's Lost. Policies for Energy-efficient Lighting. France.

Agência Nacional de Vigilância Sanitária. (2004). Resolução RDC $n^{\circ} 216$.

California Energy Commission. (2018). Voluntary California Quality Light- Emitting Diode (LED) Lamp Specification. A Voluntary Minimum Specification for "California Quality" LED Lamps.

Corazza, R. I. (2020). Gestão Ambiental e Mudanças da Estrutura Organizacional. RAE-eletrônica. 2(2)

Cury, A. (2020) Organização \& Métodos. Atlas.

Departamento de Energia dos Estados Unidos. (2018) Building Technologies Program. Solid-State lighting technology fact sheet.

Departamento de Energia dos Estados Unidos. (2017). Life-Cycle Assessment of Energy and Environmental Impacts of LED Lighting Products.

Departamento de Energia dos Estados Unidos. (2016). Energy Savings Potential of Solid-State Lighting in General Illumination Applications.

Eletrobrás. (2020). Programa Nacional de Conservação de Energia Elétrica. Pesquisa de Posse de Equipamentos e Hábitos de Uso. Ano Base 2005. Avaliação do Mercado de Eficiencia Energética do Brasil.

Estrela, C. (2018). Metodologia Científica: Ciência, Ensino, Pesquisa. Editora Artes Médicas.

Nobrega, I. (2020). PHILIPS - Tecnologias e Produtos. In: PALESTRA DA PHILIPS SOBRE LEDS PELO IPOG.

Pacific Northwest National Laboratory. (2016). Compact Fluorescent Lighting in America: Lessons Learned on the Way to the Market.

Pereira A. S. et al. (2018). Metodologia da pesquisa científica. UFSM.

Pinto, R. A. (2018). Projeto e implementação de lâmpadas, para iluminação de interiores, empregado diodos emissores de luz, LEDS. Dissertação de mestrado, Programa de pós graduação em engenharia elétrica.

Programa Ambiental das Nações Unidas. (2015). Assessment of Opportunities for Global Harmonization of Minimum Energy Performance Standards and Test Standards for Lighting Products. Collaborative Labelling and Appliance Standards Program.

Souza, A. R., Youssef, M. N., Turbino, M. L. \& Mello, F. B. (2015). Avaliação do grau de polimerização de duas resinas compostas utilizando luz halógena e dois diferentes tipos de LEDs. Revista de Pós Graduação. 12(4):474-80.

Teixeira, S. M. F. G., Oliveira, Z. M. C., Rego, J. C. \& Buscontini, T. M. B. (2018) Administração aplicada as UAN's. Atheneu. P.219.

Yin, R.K. (2015). O estudo de caso. Bookman. 